\title{
Protección Jurídica del Medio Ambiente en la Jurisprudencia de la Corte Interamericana de Derechos Humanos
}

\section{The Environmental Protection in the Jurisprudence of the Inter-American Court of Human Rights}

Submissão: 06.08.2015

Decisão Editorial: 27.08.2015

Comunicação ao autor 27.08.2015

\section{VALERIO DE OLIVEIRA MAZZUOLI}

Post-Doctor en Ciencias Jurídico-Políticas por la Universidad Clásica de Lisboa (Portugal), Doctor summa cum laude en Derecho Internacional por la Universidad Federal de Rio Grande del Sur (Brasil), Máster en Derecho por la Universidad Estadual de San Pablo (Brasil), Profesor Adjunto (Licenciatura y Máster) en la Facultad de Derecho de la Universidad Federal de Mato Grosso (Brasil), Miembro de la Sociedad Brasileña de Derecho Internacional (SBDI) y de la Asociación Brasileña de Constitucionalistas Demócratas (ABCD), Abogado y Consultor Jurídico.

\section{GUSTAVO DE FARIA MOREIRA TEIXEIRA}

Máster en Derecho Agroambiental por la Universidad Federal de Mato Grosso (Brasil), Profesor de Derecho Internacional en la Universidad de Cuiabá (Brasil), Abogado en el Estado de Mato Grosso (Brasil), Miembro del Grupo de Investigación de Derecho Internacional Público - GEDIP/UFMT.

RESUMEN: Este trabajo tiene como objetivo comprender las interrelaciones entre la protección internacional del medio ambiente y los sistemas regionales de protección de los derechos humanos, así como las aportaciones de la jurisprudencia de la Corte Interamericana de Derechos Humanos para fortalecer la protección de los derechos civiles y políticos en los casos relativos a cuestiones ambientales.

PALABRAS CLAVE: Derechos Humanos; Medio Ambiente; Sistema interamericano; Defensa por la vía refleja; Ecologización; Greening; Reverdecimiento; Convención Americana sobre Derechos Humanos.

ABSTRACT: This paper aims to understand the interrelationships between the international environmental protection and the human rights protection systems, as well as the contributions of the jurisprudence of the Inter-American Court of Human Rights to the strengthening of the civil and political rights protection in cases involving environmental issues. 
KEYWORDS: Human rights; Environment; Inter-American System; environmental protection through the reflex pathway; Ecologización; American Convention on Human Rights.

SUMARIO: 1 Introducción; 2 El sistema interamericano de derechos humanos y las cuestiones ambientales: 2.1 La protección internacional del medio ambiente y de los derechos humanos; 2.2 La ecologización de la Comisión y de la Corte Interamericanas; 3 El "escribir recto en renglones torcidos" del sistema interamericano de derechos humanos; 3.1 La defensa del medio ambiente por la vía refleja; 3.2 Efectos de la ecologización de la Corte; 4 Conclusiones; 5 Bibliografía.

SUMMARY: 1 Introduction; 2 The Inter-American human rights system and the environmental issues; 2.1 The environment and the human rights international protection; 2.2 Greening the InterAmerican Commission and the Inter-American Court; 3 "Writing straight with crooked lines" in the Inter-American human rights system; 3.1 The environmental protection through the reflex pathway technique; 3.2 Effects of the Inter-American Court greening; 4 Conclusions; 5 Bibliography.

\section{INTRODUCCIÓN}

A pesar de la falta de disposición expresa en los textos de la Declaración Americana de los Derechos y Deberes del Hombre (1948) y de la Convención Americana sobre Derechos Humanos (1969) - así también como en el Convenio Europeo de Derechos Humanos (1950) -, lo cierto es que la práctica de la Comisión y de la Corte Interamericanas de Derechos Humanos ha demostrado que en el contexto regional interamericano es posible apoyar (y, por lo tanto, proteger) las cuestiones relacionadas con el medio ambiente de manera eficaz, cuya influencia se ejemplifica por el fenómeno llamado de ecologización (greening o "reverdecimiento") del derecho internacional de los derechos humanos ${ }^{1}$.

Por tanto, el greeninges la técnica para proteger el medio ambiente en los sistemas regionales de protección que, a priori, no tienen protección específica sobre este tema. En este sentido, es necesario entender los efectos de este "reverdecimiento" de la jurisprudencia de la Corte Interamericana de Derechos Humanos y sus implicaciones para la mejora de nuestro sistema regional de protección de los derechos humanos.

\section{EL SISTEMA INTERAMERICANO DE DERECHOS HUMANOS Y LAS CUESTIONES AMBIENTALES}

Estructurado por la Carta de la Organización de los Estados Americanos, por la Declaración Americana de los Derechos y Deberes del Hombre y por la

1 Por lo tanto, es necesario desarrollar estrategias y técnicas para vincular los temas ambientales a los dispositivos de la Convención Americana, como los relacionados con el juicio justo, la libertad de expresión, los derechos de propiedad, entre otros. Sobre el tema, v. SANDS, Philippe (ed.). Greening international law. London: Earthscan Publications Limited, 1993; TEIXEIRA, Gustavo de Faria Moreira. O greening no sistema interamericano de direitos humanos. Curitiba: Juruá, 2011; y MAZZUOLI, Valerio de Oliveira \& TEIXEIRA, Gustavo de Faria Moreira. Greening the Inter-American human rights system. L'Observateur des Nations Unies, v. 33 (2013), p. 299-313. 
Convención Americana sobre Derechos Humanos, el sistema interamericano de derechos humanos, desde su creación, ha participado en los debates sobre los grandes temas del derecho internacional público.

Cabe destacar que la Declaración Americana de los Derechos y Deberes del Hombre es ocho meses más joven que la Declaración Universal de los Derechos Humanos de la ONU, del 10 de diciembre 1948; y que cuando la Carta de la OEA de 1948, en su art. 106, ha previsto un futuro Convenio orientado al establecimiento de las normas de funcionamiento de la Comisión Interamericana de Derechos Humanos, se ha también anticipado a la propia ONU en las discusiones sobre la creación de un sistema de protección de los derechos humanos ${ }^{2}$.

El dicho Convenio, la Convención Americana sobre Derechos Humanos - también conocido como Pacto de San José -, además de regular el funcionamiento de la Comisión Interamericana también creó la Corte Interamericana de Derechos Humanos, un órgano consultivo y contencioso del sistema regional interamericano.

Para los Estados Partes de la Convención que reconocen su competencia contenciosa, la Corte Interamericana actúa como el organismo responsable por el análisis de las acciones de responsabilidad internacional presentadas por los Estados Partes o por la Comisión Interamericana (con base en denuncias individuales) contra los Estados que tengan, en tesis, violado los dispositivos de la Convención Americana ${ }^{3}$. Mientras que nunca (hasta ahora) un Estado Parte ha demandado contra otro Estado Parte ante la Corte, el envío de casos a la Corte ha sido señalado por la actuación de la Comisión Interamericana, tras el análisis de las peticiones individuales que les sean presentados de conformidad con los artículos 44, 46 y 47 de la Convención. Mientras la construcción del sistema de protección de los derechos humanos de la OEA, en los años del 60 y del 70 el tema ambiental se destacó como un problema mundial importante, sobre todo después del período de la descolonización de África. Dados los dramas acerca de la degradación del medio ambiente, tanto en la Conferencia Internacional sobre el Medio Ambiente Humano (Estocolmo, 1972) como en la Conferencia de las Naciones Unidas sobre el Medio Ambiente y el Desarrollo (Río de Janeiro,

2 De los 34 miembros activos de la OEA, sólo Antigua y Barbuda, Bahamas, Belice, Canadá, Estados Unidos, Guyana, San Cristóbal y Nevis, Santa Lucía y San Vicente y las Granadinas nunca han ratificado la Convención. Para estos Estados, así como para aquellos que no reconocen la competencia de la Corte, la Comisión actúa como un órgano general que debe publicar informes anuales a la Asamblea General de la OEA sobre el desempeño de los mismos Estados en el establecimiento de políticas de los derechos humanos. V. General Information of the American Convention on Human Rights. Disponible en: http://www.oas.org/juridico/english/ Sigs/b-32.html (Último acceso el 18.04.2015). Cf. también: GOMES, Luiz Flávio \& MAZZUOLI, Valerio de Oliveira. Comentários à Convenção Americana sobre Direitos Humanos: Pacto de San José da Costa Rica. 3. ed. rev., atual e ampl. São Paulo: Revista dos Tribunais, 2010 p. 360-363; y FIX-ZAMUDIO, Hector. Protección jurídica de los derechos humanos. México: Comisión Nacional de Derechos Humanos, 1991, p.164.

3 De los 24 Estados Partes en la Convención, sólo tres - Dominica, Granada y Jamaica - aún no se someten a la jurisdicción de la Corte. 
1992), el derecho de acceso al medio ambiente sano fue insertado en la lista de las garantías fundamentales de la persona humana que deben ser protegidas ${ }^{4}$.

En 1993, la Conferencia Mundial de Derechos Humanos, celebrada en Viena, confirmó que la lógica de decir que todos los derechos humanos son universales, indivisibles e interdependientes y están relacionados entre $\mathbf{s i}^{5}$. Por lo tanto, Estocolmo, Río y Viena contribuyeron a la "globalización" ${ }^{6}$ del derecho ambiental, fenómeno consistente en una mayor comprensión de la interconexión entre los mecanismos de protección de los derechos humanos y las cuestiones ambientales.

\subsection{La PROTECCIÓN INTERNACIONAL DEL MEDIO AMBIENTE Y DE LOS DERECHOS HUMANOS}

La protección jurídica del medio ambiente, desde la Conferencia de Río-92, está estructurada por una "nueva ingeniería", consistente en acelerar la aplicación de las normas internacionales relativas al medio ambiente a través de la adopción, en los tratados "más emblemáticos", de anexos, apéndices y términos generales, que tienen el propósito de formar un amplio espacio normativo que se complementará con futuras decisiones derivadas de cumbres regulares de los Estados Partes: las Ilamadas Conferencias de las Partes o COPs ${ }^{7}$.

Estas estrategias desarrolladas por el derecho internacional del medio ambiente contemporáneo, sin embargo, no están libres de inconvenientes. Si, por un lado, las reglas ambientales son aplicadas en un ritmo más rápido, por otro, hay que señalar que esa "ingeniería" trae graves preguntas sobre la eficacia de sus decisiones, ya que los compromisos asumidos por los Estados en cuestiones ambientales constituyen reglas de soft law, o sea, son normas que, en principio, no tienen fuerza vinculante y que, por lo tanto, si no son cumplidas, no establecen sanciones directamente aplicables a los Estados ${ }^{8}$.

4 SOARES, Guido. Direito internacional do meio ambiente: emergências, obrigações e responsabilidades. São Paulo: Atlas, 2001, p. 45-49.

5 PIOVESAN, Flávia. Direitos Humanos: desafios e perspectivas contemporâneas. Revista do Instituto de Direito Constitucional e Cidadania, ano I, v. 1, abril 2005, p. 56. Cf. ainda: SOUSA SANTOS, Boaventura de. Uma concepção multicultural de direitos humanos. Revista Lua Nova, São Paulo, v. 39 (1997), p. 105-201; y HUMAN RIGHTS WATCH. World Report 1994: Events of 1993. New York, 1994, p. 17.

6 V. CANÇADO TRINDADE, Antônio Augusto. Direitos humanos e meio-ambiente: paralelo dos sistemas de proteção internacional. Porto Alegre: Sérgio Antônio Fabris Editora, 1993. p. 41-45.

7 SOARES, Guido. Dez anos após Rio-92: o cenário internacional, ao tempo da cúpula mundial sobre desenvolvimento sustentável (Joanesburgo, 2002). In: MAZZUOLI, Valerio de Oliveira \& IRIGARAY, Carlos Teodoro Hugueney (Orgs). Novas perspectivas do direito ambiental brasileiro: visões interdisciplinares. Cuiabá: Cathedral, 2009. p. 12.

8 SOARES, Guido. A proteção internacional do meio ambiente. Barueri: Manole, 2003, p. 101. V. también: MITCHELL, Ronald B. Problem structure, institutional design, and the relative effectiveness of international environmental agreements. Global Environmental Politics, v. 6, n. 3, Cambridge, August 2006, p. 72-89. 
La fragilidad o "eficacia relativa de los tratados internacionales sobre el medio ambiente" ${ }^{\prime \prime}$ demuestran que las cuestiones ambientales aún no alcanzaron su madurez en las relaciones internacionales contemporáneas. Dinah Shelton y Alexander Kiss, sin embargo, creen que esta madurez radica en el creciente establecimiento de interrelaciones entre las cuestiones ambientales y la protección de los derechos humanos ${ }^{10}$. Esto nos permite afirmar que los sistemas de protección de derechos humanos, en el final del siglo XX, pasaron por un proceso de reverdecimiento: a) la Carta Africana de Derechos Humanos y de los Pueblos (1981) y el Protocolo Adicional a la Convención Americana sobre Derechos Económicos, Sociales y Culturales (1988) insertaron dispositivos que expresamente reconocieron el derecho de vivir en un medio ambiente sano y equilibrado ${ }^{11}$; y b) el Convenio Europeo de Derechos Humanos (1950) - a pesar de la ausencia de disposiciones relativas a la protección del medio ambiente - ha sido utilizado en la jurisprudencia del Tribunal Europeo de Derechos Humanos en cuestiones ambientales ${ }^{12}$.

Aunque incipiente, el Tribunal Europeo de Derechos Humanos ha desarrollado un proceso de reverdecimiento marcado por el establecimiento de interrelaciones entre temas ambientales y los dispositivos del Convenio Europeo relacionados con los derechos a la vida, la privacidad, la propiedad y otros. Dos casos relativos a la interconexión entre la contaminación acústica causada por el aeropuerto de Heathrow, en las afueras de Londres, y la violación de disposiciones del Convenio Europeo relativos a los derechos de propiedad, la privacidad y la protección judicial efectiva, son emblemáticos: Powell y Rayner vs. Reino Unido y Hatton y otros vs. Reino Unido ${ }^{13}$.

9 MITCHELL, Ronald B. Problem structure, institutional design, and the relative effectiveness of international environmental agreements, cit., p. 72-89; y BOYLE, Alan. Human rights and the environment: a reassessment. UNEP Human Rights and Environment, 2010, p. 2-3.

10 V. KISS, Alexandre \& SHELTON, Dinah. Guide to international environmental law. Koninlijke Brill, NV, Leiden: Martinus Nijhoff, 2007; KISS, Alexandre \& SHELTON, Dinah. Judicial handbook on environmental law. Nairobi: United Nations Environment Programme, 2005; y SHELTON, Dinah. Environmental rights and Brazil's obligations in the Inter-American human rights system. George Washington International Law Review, v. 40 (2008), p. 733-777.

11 MAZZUOLI, Valerio de Oliveira. Curso de direito internacional público. 5. ed. rev., atual. e ampl. São Paulo: Editora Revista dos Tribunais, 2011. p. 1001-1003.

12 V. Tribunal Europeo, Caso Powell y Rayner vs. Reino Unido. Sentencia de 21 de febrero de 1990, Aplicación 9310/81; Caso López Ostra vs. España. Sentencia de 9 de diciembre de 1994, Aplicación 16798/90; Caso Ana Maria Guerra y otros vs. Italia. Sentencia de 19 de febrero de 1998, Aplicaciones 116/1996/735/932; Caso Hatton y otros vs. Reino Unido. Sentencia del 2 de octubre 2001, Aplicación 36022/97; Caso Moreno Gómez vs. España. Sentencia del 16 de noviembre 2004, Aplicación 4143/02; Caso Giacomelli vs. Italia. Sentencia del 2 de noviembre 2006, Aplicación 59909/00; Caso Fadeïeva vs. Rusiaa. Sentencia del 9 de junio 2005, Aplicación 55723/00; Caso Öneryildiz vs. Turquía. Sentencia del 18 de junio 2002, Aplicación 48939/99; Caso Taskin y otros vs. Turquía. Sentencia del 10 de noviembre 2004, Aplicación 46117/99; y Caso Tatar vs. Rumanía. Sentencia del 27 de enero 2009, Aplicación 67021/01. Disponible em: http://www. echr.coe.int (Último acceso el 18.04.2015).

13 Tribunal Europeo, Caso Powell y Rayner vs. Reino Unido. Sentencia de 21 de febrero de 1990, Aplicación 9310/81; y Caso Hatton y otros vs. Reino Unido. Sentencia del 2 de octubre 2001, Aplicación 36022/97. 
En 1990, el Tribunal Europeo, al analizar el caso Powell y Rayner vs. Reino Unido, a pesar de tener reconocido que el Estado británico tendría el deber de tomar medidas razonables y apropiadas para garantizar a los peticionarios la protección conferida por el art. 8.1 del Convenio Europeo (derecho a la vida privada y familiar), consideró que "la operación de un gran aeropuerto internacional es un objetivo legítimo [del Estado] y el consiguiente impacto negativo sobre el medio ambiente no puede ser totalmente eliminado"14. Sin embargo, once años después, en 2001, en el caso Hatton y otros vs. Reino Unido, también relativo al aeropuerto de Heathrow, el Tribunal Europeo concluyó que, en clara violación del art. 8.1 del Convenio Europeo, el Estado "no logró un justo equilibrio entre el bienestar económico del Reino Unido y el efectivo derecho de los peticionarios gozaren de sus hogares y de sus vidas privadas y familiares" 15 .

Es de destacar que el período de once años entre los dos casos Heathrow ${ }^{16}$ contribuyó al fortalecimiento de la comprensión de las interrelaciones entre los dispositivos del Convenio Europeo de Derechos Humanos y de las cuestiones ambientales en Europa. Después de todo, a lo largo de los años 90, sobre todo después de la Conferencia de Río-92, las cuestiones ambientales habían surgido como un tema de claro interés global.

En este contexto, otro caso decidido por el Tribunal Europeo de Derechos Humanos - Caso López Ostra vs. España, de 1994 - se ha convertido en uno de los más emblemáticos para las futuras decisiones del Tribunal. Al constatar la molestia de la familia López Ostra con las emisiones de azufre de una planta de tratamiento de agua en la ciudad española de Lorca, el Tribunal Europeo entendió que la contaminación del medio ambiente implica daños al derecho a la vida privada y familiar, y por lo tanto, el Estado tiene el deber de proporcionar un equilibrio justo entre sus medidas y el bienestar del medio ambiente y de las personas. Por entender que, en el caso analizado, el Estado no ha cumplido

14 "The operation of a major international airport pursued a legitimate aim and that the consequential negative impact on the environment could not be entirely eliminated" (Tribunal Europeo, Caso Powell y Rayner vs. Reino Unido, cit., pars. 40-42).

15 'The State failed to strike a fair balance between the United Kingdom's economic well-being and the applicants' effective enjoyment of their right to respect for their homes and their private and family lives" (Tribunal Europeo, Caso Hatton y otros vs. Reino Unido. Sentencia del 2 de octubre 2001. Aplicación 36022/97, par. 107).

16 Hay que señalar, sin embargo, que los resultados de los dos casos relacionados con el ruido del aeropuerto de Heathrow fueron similares. En Hatton y otros, frente a la decisión desfavorable para el Reino Unido, el gobierno británico apeló al Tribunal en Pleno y la Gran Sala decidió que los intentos de las autoridades locales para reducir el ruido en el aeropuerto fueron suficientes para no haber violación del derecho a la intimidad de los demandantes. El Tribunal Europeo es formado por: a) los Comités, formados por tres jueces responsables por el análisis de admisibilidad de las peticiones presentadas a la Corte; b) las Secciones (las Salas), integradas por siete jueces encargados por el examen de la admisibilidad y del fondo de una petición; y c) el Pleno del Tribunal (la Gran Sala), integrado por diecisiete jueces responsables por el examen de las remisiónes de un asunto pendiente ante una Sala sobre una cuestión grave relativa a la interpretación del Convenio o de sus Protocolos (arts. 30 y 43, $\S 3^{\circ}$ Del Covenio Europeo). V. MAZZUOLI, Valerio de Oliveira. Curso de direito internacional público, cit., p. 913-915; y GOMES, Carla Amado. Textos dispersos de direito do ambiente. Lisboa: Associação Acadêmica da Faculdade de Direito, v. III, 2010. p. 183. 
con su deber de conciliar los intereses colectivos con los intereses particulares de los vecinos de la planta de tratamiento de residuos, el Tribunal condenó a España a pagar 40.000 pesetas por los daños a la salud y la calidad de vida de la familia López Ostra ${ }^{17}$.

López Ostra vs. España también dio lugar a una segunda fase del reverdecimiento del Tribunal Europeo: el aumento de las interrelaciones entre los casos que involucran cuestiones ambientales y la protección de los dispositivos del Convenio Europeo de Derechos Humanos. En Anna Maria Guerra y otros vs. Italia (1998) el Tribunal Europeo concluyó que al no proporcionar la información necesaria sobre una fuga de productos químicos de la fábrica en Manfredonia, las autoridades locales habían cometido una violación al art. 10 del Convenio Europeo sobre el derecho a la libertad de expresión y el acceso a la información. En 2004, el derecho a la vida privada y familiar, garantizado por el art. 8.1 de la Convenion Europeo, fue una vez más interrelacionado con un tema ambiental, esta vez en el caso Moreno Gómez vs. España, sobre la contaminación acústica causada por un club nocturno construido sobre la licencia ilegal a las afueras de Valencia ${ }^{18}$.

A lo largo de la primera década de este siglo, otros dos casos han sido emblemáticos a la ecologización del Convenio Europeo: a) Öneryildiz vs. Turquía (2002), sobre violaciones de los arts. 2, 8 y 13 del Convenio Europeo, relativos respectivamente a los derechos a la vida, a la vida privada y familiar y a la protección judicial efectiva de las víctimas de una explosión de gas metano en el relleno sanitario de Umraniye (Estambul) ${ }^{19}$; y b) Tatar vs. Rumanía (2009), sobre un desastre ecológico resultante de las instalaciones de descarga de una mina de oro en Río Sasar, donde el Tribunal Europeo concluyó que el Estado rumano había violado el Convenio al no proporcionar un deber de prevención e información sobre los riesgos de instalaciones de mina de oro $^{20}$.

Las diversas situaciones en que la defensa del medio ambiente por la vía refleja se hizo presente en el Tribunal Europeo de Derechos Humanos - del interior de Rumanía al aeropuerto de Heathrow, de la molestia de discotecas en el centro de Valencia a tragedias de un vertedero de basura en las afueras

17 Tribunal Europeo, Caso López Ostra vs. España. Sentencia de 9 de diciembre de 1994, Aplicación 16798/90, pars. 51 y 58.

18 V. Caso Ana Maria Guerra y otros vs. Italia. Sentencia de 19 de febrero de 1998, Aplicaciones 116/1996/735/932; y Caso Moreno Gómez vs. España. Sentencia del 16 de noviembre 2004, Aplicación 4143/02.

19 V. Caso Öneryildiz vs. Turquía. Sentencia del 18 de junio 2002, Aplicación 48939/99.

20 "La Cour rappelle qu'en droit roumain le droit à un environnement sain est un principe ayant valeur constitutionnelle. Ce principe a été repris par la loi no 137/1995 sur la protection de l'environnement, qui était en vigueur à l'époque des faits (voir pp. 16-17, a et b). Par ailleurs, le principe de précaution recommande aux États de ne pas retarder l'adoption de mesures effectives et proportionnées visant à prévenir un risque de dommages graves et irréversibles à l'environnement en l'absence de certitude scientifique où technique (voir $p$. 27, h)" (Tribunal Europeo, Caso Tatar vs. Rumanía. Sentencia del 27 de enero 2009, Aplicación 67021/01, par. 109). 
de Estambul - muestran que el tema del medio ambiente tiene la potencial vocación de servir como parámetro para otros sistemas regionales de protección de los derechos humanos ${ }^{21}$.

En este sentido, merece ser estudiado como la Comisión Interamericana y la Corte Interamericana de Derechos Humanos han estado lidiando con cuestiones ambientales, así como el papel de sus posiciones en lo reverdecimiento o ecologización cada vez más eficaz de la Convención Americana sobre Derechos Humanos.

\subsection{La ecologización de la Comisión y de la Corte Interamericanas}

En comparación con el sistema europeo, la ecologización del sistema interamericano ha pasado lejos de cuestiones tales como la contaminación acústica causada por un club nocturno o un aeropuerto. De hecho, la gran mayoría de los casos ambientales del sistema interamericano surgen del uso constante de las zonas forestales y de las zonas rurales en la búsqueda por materias primas, alimentos, agua, combustible y hacia el uso zonas de disposición de basura. En este contexto, los más vulnerables a la desregulada explotación económica de los recursos naturales han sido los indígenas, los pueblos cimarrones y las comunidades campesinas de las Américas ${ }^{22}$.

Este hallazgo es confirmado por el informe de la Secretaría General de la OEA sobre Derechos Humanos y Medio Ambiente, de 4 de abril de 2002 ${ }^{23}$, que destaca dos ejemplos de protección del medio ambiente en el sistema interamericano: a) La Resolución no 12/85 del pueblo Yanomami vs. Brasil ${ }^{24}$, que se ocupa de las interrelaciones entre la construcción de una carretera en una zona del territorio amazónico habitada por la etnia Yanomami y el rápido proceso de violación de los derechos a la vida, la salud, la libertad, la seguridad y el derecho de residencia del grupo indígena afectado; b) y el caso de la Comunidad Indígena Awas Tingni Mayagna (Sumo) vs. Nicaragua ${ }^{25}$, sobre la concesión irregular de madera en tierras indígenas. Estos son los primeros casos de cuestiones ambientales examinados por la Comisión y por la Corte Interamericana de Derechos Humanos, respectivamente.

21 V. GOMES, Carla Amado. Textos dispersos de direito do ambiente, cit., p. 163-205.

22 Sobre el progreso del poder económico en las áreas de los pueblos indígenas y tradicionales, v. GARFIELD, Seth. A nationalist environment: indians, nature and the construction of the Xingu National Park in Brazil. Luso-Brazilian Review, v. 41, n. 1 (2004), p. 139-167; SHIVA, Vandana. Biopirataria: a pilhagem do conhecimento. Trad. Laura Cardellini Barbosa de Oliveira. Petrópolis: Vozes, 2001, p. 23-29; y VARVARIGOS, Dimitrios. Environmental degradation, longevity and the dynamics of economic development. Environmental and Resource Economics, v. 46, issue 1 (2010), p. 59-73.

23 En el cumplimiento de AG/Res. 1819 (XXXI-O/01), aprobada en la tercera sesión plenaria de la OEA, celebrada el 5 de junio 2001. V. MAZZUOLI, Valerio de Oliveira. Curso de direito internacional público, p. 1001.

24 Resolución 12/85, Caso no. 7615 (Brasil), 5 de marzo de 1985, en Informe Anual de la CIDH 1984-85, OEA/ Ser.L/V/II.66, Doc. 10 rev.1, 1 de octubre de 1985, 24, 31 (Caso Yanomami).

25 Caso de la Comunidad Mayagna (Sumo) Awas Tingni vs. Nicaragua. Sentencia de 31 de agosto de 2001. Serie C, $n^{\circ}$ 79. Disponible en: http://www.corteidh.or.cr/casos.cfm (Último acceso el 18.04.2015). 
Después de la Resolución no 12/85, la Comisión tuvo que hacer frente, en particular, con otros ocho casos relacionados con temas ambientales. Tres de ellos - en eventos similares en Chile, Panamá y Brasil - involucran la construcción de represas hidroeléctricas en zonas indígenas y/o tradicionales sin el consentimiento de las comunidades afectadas y las consecuentes violaciones de disposiciones de la Convención Americana en relación con la garantía a la vida, la dignidad humana y la protección judicial efectiva ${ }^{26}$.

En este sentido, en el caso de Las comunidades indígenas Mayas de Toledo vs. Belice, la Comisión Interamericana estableció interrelaciones entre la concesión por el Estado de las tierras indígenas a la tala sin el consentimiento previo de los grupos étnicos afectados, con violaciones de los derechos de propiedad de los pueblos tradicionales ${ }^{27}$. A su vez, en el caso San Mateo de Huanchor vs. Perú, donde se utilizó un minero en las afueras de las comunidades rurales como un vertedero de residuos tóxicos, y en el análisis de la Solicitud Inicial no 1413/05 del Pueblo Inuit contra los Estados Unidos sobre las responsabilidades del gobierno de E.E.U.U en relación con los impactos del calentamiento global en el modo de vida de los esquimales, la Comisión se enfrentó a situaciones relacionadas con la degradación del medio ambiente y sus consecuencias sobre los medios de vida de los pueblos tradicionales, en particular en el acceso de estos pueblos a los derechos a la vida, la libertad, la seguridad, la residencia y el acceso a la cultura ${ }^{28}$.

De nueve casos importantes sobre cuestiones ambientales discutidos o en análisis por la Comisión, sólo dos - Informe n 84/03 del Parque Natural Metropolitano de Panamá, sobre el supuesto daño ambiental causado por la construcción de una carretera en una reserva ambiental, y Comunidad de La Oroya vs. Perú, en relación con hechos perjudiciales de la contaminación del aire de un complejo metalúrgico en la población de 30.000 habitantes de La Oroya, ubicada a $175 \mathrm{~km}$ de Lima - no han tratado sobre temas relacionados con los pueblos indígenas o con comunidades tradicionales ${ }^{29}$.

26 Sobre casos de cuestiones ambientales analizados por la Comisión Interamericana, v. TEIXEIRA, Gustavo de Faria Moreira, 0 greening no sistema interamericano de direitos humanos, cit., p. 163-227. Cf. CIDH. Informe n 30/2004, Solución Amistosa Mercedes Julia Huentes Beroiza, 11 de marzo de 2004; CIDH. Informe no 40/2004, Caso de Las comunidades indígenas Mayas de Toledo vs. Belice, 12 de octubre de 2004; y Medida Cautelar MC-382/10, en favor de las comunidades tradicionales de la cuenca del río Xingu, Pará, Brasil, el 11 de noviembre, 2009.

$27 \mathrm{CIDH}$. Informe n 40/2004, Caso de Las comunidades indígenas Mayas de Toledo vs. Belice, 12 de octubre de 2004.

28 V. CIDH. CIDH. Informe n 69/2004, Caso San Mateo Huanchorvs. Peru, OEA/Ser.L/V/II.122, Doc. 5, rev.1, octubre de 2004; y Inuit People Petition 1413/2005 vs. theUnitedStates, December 7, 2005. Cf. OSOFSKY Hari M. Inuit petition as a bridge? Beyon dialectics of climate change and indigenous people's rights. American Indian Law Review. v. 31 (2007), p. 675-698.

29 V. CIDH. Informe no 84/2003, Parque Natural Metropolitano de Panamá, 22 de octubre 2003; y Informe no 76/25009, CasoComunidad de La Oroya vs. Perú, 5 de agosto 2009. Cf. SPILLER, Paula. The La Oroya case: the relationship between environmental degradation and human rights violation. Human rights brief. V. 18, issue 1, Washington D.C: Washington College of Law, fall 2010, p. 19 y 22; y CEDERSTAV, Anna K; BARANDIARÁN G., Alberto. La Oroya cannot wait. Lima: Sociedad Peruana de Derecho Ambiental, 2002. 
La Corte Interamericana, a su vez, después del análisis del caso Comunidad Mayagna (Sumo) Awas Tingni vs. Nicaragua, se enfrentó con otros seis casos sobre temas ambientales, y de éstos, cinco están relacionados con violaciones de derechos de los pueblos tradicionales de las Américas; un caso - caso Pueblo Indígena Kichwa de Sarayacu vs. Equador - es vinculado a la concesión estatal de tierras indígenas para la exploración petrolera sin el consentimiento de los Kichwas ${ }^{30}$; y los otros cuatro son relativos a los impactos adversos de la falta de demarcación de las tierras de los pueblos indígenas y cimarrones en Paraguay y en Surinam en los siguientes episodios: a) caso Moiwana vs. Surinam; b) caso Comunidad Indígena Yakye Axa vs. Paraguay; c) caso Comunidad Indígena Sawhoyamaxa vs. Paraguai; y d) caso Pueblo de Saramaka vs. Surinam ${ }^{31}$. Estos casos revelan posiciones que, inevitablemente, se desarrollaron a partir del análisis de casos que difícilmente se pueden abordar de manera rutinaria en un sistema de protección que no se inserta en las especificidades regionales de las Américas.

A modo de ejemplo, los casos de violaciones de derechos de los pueblos indígenas y tradicionales Ilaman la atención. En éstos, tanto la Comisión como la Corte Interamericana han consolidado el entendimiento de que el concepto del derecho a la propiedad privada previsto en el art. 21 de la Convención Americana no se limita al concepto occidental - que es en particular marcado por el ejercicio de derechos mercadológicos, como los derechos a la alienación, la división, la exclusión, la hipoteca y el uso y el disfrute de un bien -, pero también cubre los elementos que componen la propiedad comunal de los pueblos tradicionales. ${ }^{32}$ Reconociendo el concepto indígena de la propiedad, la Corte fortalece una óptica en la que el derecho de propiedad garantizado por la Convención Americana también se ejerce por asegurar a los pueblos tradicionales el uso de los recursos naturales en sus tierras como una forma de mantener sus hábitos culturales, como la religión, las prácticas agrícolas, la caza, la pesca y el modo de vida de su comunidad. ${ }^{33}$

30 Caso Pueblo Indígena Kichwa de Sarayaku vs. Ecuador, Sentencia de 27 de junio de 2013. Série C, n 245.

31 Sobre casos de cuestiones ambientales analizados por la Corte Interamericana de Derechos Humanos, v. TEIXEIRA, Gustavo de Faria Moreira, O greeningno sistema interamericano de direitos humanos, cit., p. 229-286. Cf. Caso Moiwana vs. Surinam. Sentencia del 15 de junio 2005. Serie C, no 124; CasoComunidad Indígena Yakye Axa vs. Paraguay. Sentencia 17 de junio 2005. Serie C, $n^{\circ} 125$; Caso Comunidad Indígena Sawhoyamaxa. Paraguay. Sentencia de 29 de marzo, 2006. Serie C, $n^{\circ} 146$; y Caso Pueblo de Saramaka vs. Surinam. Sentencia del 28 de noviembre 2007. Serie C, n 172.

32 V. ANKERSEN, Thomas T. \& RUPPERT, Thomas K. Defending the polygon: the emerging human right to communal property. Oklahoma Law Review, v. 59, n. 4 (2006), p. 716-719; MAGRAW, Daniel Barstow \& BAKER, Lauren. Globalization and communities: community-based property rights and prior informed consent. Denver Journal of International Law and Policy, v. 35, ns 3 y 4, 2007/2008, p. 416-418; KISS, Alexandre \& SHELTON, Dinah. Judicial handbook on environmental law., cit., p. 105; SHELTON, Dinah. Environmental Rights and Brazil's Obligations in the Inter-American Human Rights System, cit., p. 759-760; y SHELTON, Dinah. Human rights, health and environmental protection: in Law and practice. Health and human rights working paper series $n^{\circ} 1$. Ginebra: World Health Organization, 2002, p. 16.

33 "Para las comunidades indígenas la relación con la tierra no es meramente una cuestión de posesión y producción sino un elemento material y espiritual del que deben gozar plenamente, inclusive para preservar su 
Desde la perspectiva indígena de propiedad - inherente al fuerte enlace de los pueblos indígenas con la naturaleza - las decisiones de la Corte han demostrado que los dispositivos de la Convención Americana tienen un amplio alcance. De hecho, en el caso Moiwana vs. Surinam, después de señalar que los miembros de una comunidad cimarrona (expulsados de sus tierras tradicionales después de una masacre organizada por el ejército surinamés) vivían bajo un intenso sufrimiento psicológico debido a la lejanía de sus hábitos culturales y religiosos, intensamente relacionados con el contacto con la naturaleza y sus tierras tradicionales, la Corte consideró que las disposiciones de la Convención Americana sobre derechos de propiedad, de circulación y residencia, y libertad de conciencia y de religión estaban siendo violados ${ }^{34}$.

A su vez, en el caso Yakye Axa vs. Paraguay la Corte fortalece el entendimiento de que el derecho a la vida no se limita al derecho a la supervivencia en sí, sino que se extiende a la promoción de una vida con dignidad, ejercida de forma completa con el acceso a los beneficios de la cultura, la salud, la alimentación, educación y un medio ambiente sano. En Sawhoyamaxa vs. Paraguay y en Saramaka vs. Surinam, la ineficacia o la ausencia de mecanismos judiciales capaces de asegurar a los pueblos tradicionales el derecho de propiedad sobre sus tierras ancestrales hicieron la Corte considerar que los Estados demandados estaban violando el art. 3 de la Convención Americana, relativo a la protección del derecho al reconocimiento de la personalidad jurídica ${ }^{35}$.

En la Corte Interamericana, la única decisión (aún) en torno a cuestiones ambientales no relacionadas con los grupos indígenas o tradicionales es el caso Claude Reyes y otros vs. Chile, sobre la negativa del Estado de Chile para proporcionar datos sobre un proyecto de deforestación a tres ciudadanos chilenos. En este caso el derecho a la libertad de expresión prevista en el art. 13 de la Convención tuvo su alcance ampliado a afirmar la necesidad de garantizar el acceso a la información, en particular sobre temas ambientales ${ }^{36}$.

\section{EL “ESCRIBIR RECTO EN RENGLONES TORCIDOS” DEL SISTEMA INTERAMERICANO DE DERECHOS HUMANOS}

Hay que señalar que la incipiente (aunque creciente) presentación de cuestiones ambientales en el sistema interamericano sólo es posible a través de

legado cultural y transmitirlo a las generaciones futuras" (Corte IDH. Caso de la Comunidad Mayagna (Sumo) Awas Tingni vs. Nicaragua, cit., par. 149).

34 V. Caso Moiwana vs. Surinam, cit., par. 87.

35 V. ANKERSEN, Thomas T. \& RUPPERT, Thomas K. Defending the polygon: the emerging human right to communal property, cit., p. 726-732; y SHELTON, Dinah. Environmental Rights and Brazil's Obligations in the Inter-American Human Rights System, cit., p. 764-770; Cf. Comunidad IndígenaYakyeAxa vs. Paraguay, cit., pars. 161-162; y 242; y Caso Comunidad Indígena Sawhoyamaxa vs. Paraguay, cit., par. 248; y Caso Pueblo de Saramakavs. Surinam, cit., pars. 167 y 175.

36 V.Caso Claude Reyes y otros vs. Chile.Sentencia del 19 de septiembre 2006. Serie C, no 151. 
la estricta observancia de la Convención Americana sobre Derechos Humanos. El Informe no 84/03 del Parque Metropolitano de Panamá es un gran ejemplo de que el incumplimiento de las disposiciones de la Convención Americana implica fatalmente la inadmisibilidad de la petición.

El 11 de agosto de 1995 el Sr. Rodrigo Noriega envió la petición no 11.533 a la Comisión Interamericana, sobre presuntas violaciones de interés de "grupos ecológicos, cívicos y científicos" y de los derechos de propiedad de los "ciudadanos de la República de Panamá" como consecuencia de la autorización del gobierno de construir una carretera a través del Parque Metropolitano, un área de reserva ecológica, científica y cultural de Panamá . Mediante el análisis de la petición, la Comisión señaló que el art. 44 de la Convención establece que "cualquier persona o grupo de personas" tiene la autoridad para presentar una petición ante la Comisión. A pesar, sin embargo, de la expresión "cualquier persona o grupo de personas" ser amplia, la jurisprudencia interamericana establece que una petición es admisible cuando hay víctimas concretas, individualizadas y determinadas, no siendo admitido peticiones "en abstracto" $^{\prime 37}$. De hecho, la naturaleza de la víctima se requiere que esta sea un individuo, ya que la Comisión no tiene jurisdicción sobre los derechos de las personas jurídicas de derecho privado o público ${ }^{38}$.

Teniendo en cuenta estas consideraciones, la Comisión señaló que la Petición $\mathrm{n}^{\mathrm{o}}$ 11.533, representando "a todos los ciudadanos de Panamá", no cumplió con el requisito de elegibilidad para la determinación de los individuos o grupos. Al afirmar que las obras afectan a "grupos ecológicos, cívicos y científicos", la Petición no 11.533 introdujo personas jurídicas - y no individuos - en la lista de víctimas, en consecuente violación a la Convención Americana. Por lo tanto, la Comisión señaló su ausencia de competencia ratione personae para analizar el caso del Parque Natural Metropolitano ${ }^{39}$.

El resultado del caso Parque Metropolitano, según Dinah Shelton, deja la lección de que cuánto más amplio o abstracto es la indicación de las víctimas de violaciones de derecho garantizados por la Convención Americana - y esto ocurre comúnmente en cuestiones de daños ambientales -, menores son las posibilidades de admisibilidad de un caso ante la Comisión Interamericana ${ }^{40}$.

En otras palabras, la protección del medio ambiente en el sistema interamericano ocurre por una vía refleja, indirecta, ejercida por la estricta observancia de los dispositivos de la Convención Americana; surge del

$37 \mathrm{CIDH}$. Informe $\mathrm{n}^{\circ} 84 / 03$, cit., pars. 28-32.

$38 \mathrm{CIDH}$. Informe $\mathrm{n}^{\circ} 84 / 03$, cit., par. 33.

$39 \mathrm{CIDH}$. Informe n० 84/03, cit., pars. 34-37.

40 "Unfortunately, the Commission's analysis suggests that the more widespread the violations - which can occur in many contexts where environmental harm is the origin of the complaint - the less likely the Commission will find the complaint admissible" (SHELTON, Dinah. Environmental rights and Brazil's obligations in the Inter-American human rights system, cit., p. 775). 
ejercicio de "escribir recto en renglones torcidos" 41 , es decir, no surge de la preocupación ambiental en sí mismo, pero de la necesidad pragmática de proteger las disposiciones de la Convención Americana, como, por ejemplo, las relacionadas con las garantías judiciales (art. 8.1), la libertad de religión (art. 12) y de expresión (art. 13), o incluso el derecho a la propiedad (art. 21), sino también a las disposiciones relativas a los requisitos de admisibilidad de un caso en el sistema interamericano (arts. 46 y 47 ).

\subsection{La DEFENSA DEL MEDIO AMBIENTE POR LA Vía REFLEAa}

La técnica de la protección del medio ambiente por la vía refleja se desarrolla a partir de la idea de que en el marco del actual derecho internacional del medio ambiente la protección de la biosfera es eficaz a través de la indirecta y necesaria protección de los seres humanos ${ }^{42}$.

La protección del medio ambiente por la vía refleja se deriva del análisis de tres concepciones sobre el medio ambiente observadas por Alan Boyle:

a) la primera utiliza los mecanismos de protección de los derechos civiles y políticos existentes como herramienta en apoyo a las causas ambientales, especialmente cuando se trata de los derechos a la información, a la participación y al desarrollo de medidas legales de protección jurídica;

b) el segundo enfoque vincula el derecho de acceso a un ambiente "saludable", "equilibrado" y "decente" a la lista de los derechos económicos, sociales y culturales, como los derechos al desarrollo o el acceso a la salud; $y$

c) la tercera percepción considera "la calidad ambiental", por si mismo, un derecho colectivo, de solidaridad, capaz de garantizar más a la comunidad que a los individuos el derecho de determinar cómo los bienes ambientales deben ser protegidos y manejados ${ }^{43}$.

Según Alan Boyle, a pesar de que las tres percepciones siguen siendo válidas, la idea de que el medio ambiente en sí mismo ya se establece como un derecho a ser protegido se demuestra ineficaz a nivel internacional, porque los llamados derechos de solidaridad están envueltos en un sistema de control muy

41 Según Carla Amado Gomes la protección ambiental en los sistema regionales de derechos humanos es un ejercício de "escrever verde por linhas tortas" (Textos dispersos de direito do ambiente, v. III, cit., p. 163-205).

42 V. KISS, Alexandre \& SHELTON, Dinah. Judicial handbook on environmental law, cit., p. 30-31.

43 "First, existing civil and political rights can be used to give individuals, groups and NGOs access to environmental information, judicial remedies and political processes. [...] A second possibility is to treat a decent, healthy or sound environment as an economic or social right, comparable to those whose progressive attainment is promoted by the 1966 UN Covenant on Economic Social and Cultural Rights. [...] The third option would treat environmental quality as a collective or solidarity right, giving communities ('peoples') rather than individuals a right to determine how their environment and natural resources should be protected and managed" (BOYLE, Alan. Human rights and the environment: a reassessment, cit., p. 1). 
débil. Este diseño hace que se compruebe que "hay derechos que simplemente no pueden ser reclamados ante un tribunal por sus sujetos activos (titulares)" ${ }^{\prime 4}$. Por lo tanto, para que una situación de este tipo no venga a causar daño la protección al medio ambiente, el enfoque más adecuado es lo que busca un reverdecimiento (o un greening) de los ya existentes mecanismos de protección de los derechos civiles, políticos, económicos, sociales y culturales ${ }^{45}$.

Así, el derecho al medio ambiente adquiere dos dimensiones: una individual y una colectiva. A nivel individual, lo que garantiza un ambiente sano está presente en las relaciones verticales y horizontales. Por garantías ambientales obligatorias en las relaciones verticales debemos entender las que implican las relaciones jurídicas entre el Estado y los individuos. Por lo tanto, la relación vertical implica la interacción de un individuo o grupos de individuos con instrumentos jurídicos estatales que se ocupan de la protección de los recursos naturales y de garantizar los derechos civiles y políticos, como el acceso a la información y participación en la gestión de los bienes ambientales ${ }^{46}$.

En el plano horizontal, la protección del medio ambiente surge de la aplicación del "Drittwirkung" o "thirdpartyeffect". El Drittwirkung implica la efectividad de los derechos fundamentales, no sólo en la relación entre los individuos y el Estado, sino también en las relaciones jurídicas que involucran sólo a los individuos. El reverdecimiento o la "ecologización" de los derechos civiles y políticos, por lo tanto, implica en un Drittwirkung del medio ambiente, es decir, en lo que Cançado Trindade explica cómo el "Drittwirkung de la doctrina alemana, aplicada a la protección de los derechos humanos y la protección del medio ambiente" , capaz de imponer, en las relaciones entre los individuos de naturaleza contractual, laboral o de derecho civil, la observancia de normas que garanticen a las partes implicadas el derecho fundamental de contacto con un medio ambiente sano ${ }^{47}$.

En la dimensión colectiva de la protección del medio ambiente se convierte en bien común, resultado de la ecologización de los derechos económicos, sociales y culturales. Esta dimensión se ha implicado en la tendencia de proteger

44 CANÇADO TRINDADE, Antônio Augusto. Os direitos humanos e o meio ambiente. In: SYMONIDES, Janusz (Org). Direitos humanos: novas dimensões e desafios. Brasília: UNESCO Brasil/Secretaria Especial dos Direitos Humanos, 2003, p. 187.

45 "The first approach is essentially anthropocentric [...] it amounts to a 'greening' of human rights law, rather than a law of environmental rights. The second comes closer to seeing the environment as a good in its own right, but nevertheless one that will always be vulnerable to tradeoffs against other similarly privileged but competing objectives, including the right to economic development. The third approach is the most contested. Not all human rights lawyers favour the recognition of third generation rights, arguing that they devalue the concept of human rights, and divert attention from the need to implement existing civil, political, economic and social rights fully" (BOYLE, Alan. Human Rights and the environment: a reassessment, cit., p. 1-2).

46 V. CANÇADO TRINDADE, Antônio Augusto. Os direitos humanos e o meio ambiente, cit., p. 187-188.

47 V. CANÇADO TRINDADE, Antônio Augusto. Idem, p. 187-188. Cf. también: ENGLE, Eric. Third party effect of fundamental rights (Drittwirkung). Hanse Law Review, v. 5, n. 2, Bremen: Hanse Law School, p. 165-173. 
a los grupos y comunidades que se encuentran en vulnerabilidad debido a la degradación del medio ambiente ${ }^{48}$.

En el sistema interamericano, la defensa por la via refleja está garantizada por el art. 19(6) del Protocolo Adicional a la Convención Americana sobre Derechos Económicos, Sociales y Culturales - el Protocolo de San Salvador, de 17 de noviembre de 1988 -, que establece que la presentación de casos relativos a violaciones de sus dispositivos ${ }^{49}$ al sistema interamericano sólo es posible por la demostración de sus interconexiones con las disposiciones de la Convención Americana ${ }^{50}$.

Así, para que cuestiones relativas al derecho de acceso a un ambiente sano, protegido por el art. 11 del Protocolo de San Salvador, sean analizadas por la Comisión y por la Corte Interamericanas, es necesario demostrar que el presunto caso de degradación del medio ambiente implica en violaciones de los dispositivos de la Convención Americana.

Las disposiciones de la Convención que más se relacionan estrechamente a las cuestiones ambientales, son: a) el derecho al reconocimiento de la

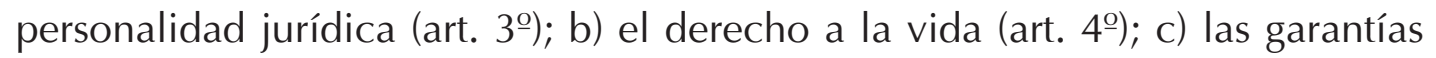
judiciales (art. 8.1); d) la tutela judicial efectiva (art. 25); y e) el principio de igualdad ante la ley y la prohibición de cualquier índole de discriminación (art. $1.1)^{51}$.

\subsection{Efectos de la ecologización de La Corte}

La estricta observancia de los dispositivos de la Convención Americana para que un problema ambiental sea amparado por el sistema interamericano es de hecho un obstáculo a la protección internacional del medio ambiente. Sin embargo, es necesario destacar que dentro del actual marco normativo internacional, marcado por la fragilidad o por normas de "eficacia relativa" que protegen el medio ambiente por sí mismo, la técnica de la protección ambiental por la vía refleja o indirecta parece ser una vía importante de maduración y mejora de los mecanismos destinados a defender no sólo el medio ambiente sino también los derechos humanos ${ }^{52}$, que son, sin duda, dos de los temas más importantes de la posmodernidad jurídica.

48 V.CANÇADO TRINDADE, Antônio Augusto. Direitos humanos e meio-ambiente..., cit., p. 89-112.

49 Excepto en casos de violaciones a el párrafo "a" del art. 8 y del art. 13 del Protocolo (relativos, respectivamente, a los derechos sindicales y al derecho a la educación).

50 "Se um peticionário simplesmente sustenta que uma decisão por um tribunal nacional foi errada em termos da legislação interna, sem alegar violação da Convenção, a petição será rechaçada" (RODRÍGUEZ-PINZÓN, Diego \& MARTín, Claudia. A proibição de tortura e maus-tratos pelo sistema interamericano: um manual para vítimas e seus defensores. Trad: Regina Vargas. Ginebra: World Organization Against Torture, 2006, p. 68-69).

51 MAZZUOLI, Valerio de Oliveira \&TEIXEIRA, Gustavo de Faria Moreira. Greening the Inter-American human rights system, cit., p. 309.

52 CANÇADO TRINDADE, Antônio Augusto. Os direitos humanos e o meio ambiente, cit., p. 191. 
En el sistema interamericano, el hecho de que la mayoría de los casos ambientales se ocupan de violaciones de los derechos de las comunidades indígenas y tradicionales, llevó a la adopción por la Comisión y por la Corte Interamericana de posiciones que difícilmente se pueden abordar de manera rutinaria en un sistema de protección de los derechos humanos que no es insertada en las especificidades regionales de las Américas. Por cierto que percibimos en estas posiciones la presencia cada vez más frecuente de los elementos del "derecho posmoderno", como el reconocimiento del pluralismo, de la comunicación intercultural, de la valoración de los sentimientos humanos y de las normas narrativas ${ }^{53}$.

El reconocimiento del pluralismo se verifica desde el entendimiento de que el alcance de los dispositivos Convención Americana no se limita a los valores culturales occidentales. A modo de ejemplo, la Corte Interamericana, en el caso Awas Tingni vs. Nicaragua, al concluir que los derechos de propiedad de comunidades indígenas fueron violados por los daños ambientales causados por la explotación forestal irregular de sus tierras tradicionales, se ha demostrado que la protección de los derechos de propiedad garantizada por el art. 21 de la Convención Americana se extiende (a) a la percepción occidental de los derechos de propiedad, de forma similar a una especie de "commodity de mercado" 54 relacionada con el derecho de un individuo "usar, gozar y disponer de sus bienes", y también (b) al concepto de propiedad comunal de los pueblos indígenas, ejercido para garantizar a estos pueblos el uso de los recursos naturales de sus tierras tradicionales como una forma de mantener sus hábitos culturales, como la religión, las prácticas agrícolas, la caza, la pesca y las formas la vida de sus comunidades ${ }^{55}$.

53 JAYME, Erik. Identité culturelle et intégration: le droit international privé post-moderne. Recueil des Cours, v. 251 (1995), p. 9-267; y MAZZUOLI, Valerio de Oliveira. Direito internacional privado: curso elementar. Rio de Janeiro: Forense, 2015, p. 199-212.

54 ANKERSEN, Thomas T. \& RUPPERT, Thomas K. Defending the polygon: the emerging human right to communal property, cit., p. 684.

55 "Entre los indígenas existe una tradición comunitaria sobre una forma comunal de la propiedad colectiva de la tierra, en el sentido de que la pertenencia de ésta no se centra en un individuo sino en el grupo y su comunidad. Los indígenas por el hecho de su propia existencia tienen derecho a vivir libremente en sus propios territorios; la estrecha relación que los indígenas mantienen con la tierra debe de ser reconocida y comprendida como la base fundamental de sus culturas, su vida espiritual, su integridad y su supervivencia económica. Para las comunidades indígenas la relación con la tierra no es meramente una cuestión de posesión y producción sino un elemento material y espiritual del que deben gozar plenamente, inclusive para preservar su legado cultural y transmitirlo a las generaciones futuras" (Caso ComunidadeMayagna (Sumo) Awas Tingnivs. Nicaragua, cit., par. 149). Cf. MAGRAW, Daniel Barstow \& BAKER, Lauren. Globalization and communities: community-based property rights and prior informed consent, cit., p. 416-418. Cf. también: GARFIELD, Seth. A nationalist environment: indians, nature and the construction of the Xingu National Park in Brazil, cit., p. 146-147; MAYZOLER, Marcel \& ROUDART, Laurence. História das agriculturas no mundo: do neolítico à cise contemporânea. Trad. Cláudia F. Fallhuh Balduino Ferreira. São Paulo: Editora Unesp; Brasília: NEAD, 2010,p. 245-250; SHIVA, Vandana. Biopirataria: a pilhagem do conhecimento, cit., p. 18-21; y SHELTON, Dinah. Environmental Rights and Brazil's Obligations in the Inter-American Human Rights System. George Washington International Law Review, v. 40 (2008), p. 756-768. 
La Corte Interamericana también ha expresado su preocupación por el llamado "retorno del sentimiento humano", porque en el caso Moiwana vs. Surinam, la constatación de que el sufrimiento psicológico de los cimarrones que estaban lejos de sus tierras tradicionales - porque se sentían perturbados por la "ira de los espíritus de los muertos" de una masacre promovida por las fuerzas armadas de Surinam contra la comunidad N'djuka - hizo a la Corte desarrollar el concepto de daño "espiritual", debido a las violaciones de dispositivos de la Convención Americana relacionados con el derecho a la integridad personal, garantías judiciales y la protección, los derechos de propiedad y el derecho de circulación y residencia. Más que una expresión, la preocupación por el "daño espiritual" causado a los demás indica que el entendimiento o comprensión de los sentimientos humanos es un elemento importante en la búsqueda de la mejor solución a un conflicto judicial ${ }^{56}$.

Al analizar el caso Claude Reyes y otros vs. Chile, sobre la negativa del gobierno de Chile para proporcionar información sobre un proyecto de deforestación a cuatro ciudadanos, la Corte reforzó el entendimiento de que el término "libertad de pensamiento y de expresión", contenido en el art. 13 de la Convención Americana, también incluye el "derecho y la libertad de buscar, recibir y difundir informaciones e ideas de toda índole" ${ }^{27}$. Este punto de vista se basa en particular en la conclusión de que el Estado chileno debe observar el principio 10 de la Declaración de Río sobre el Medio Ambiente y el Desarrollo, que asegura a "todo individuo" el "acceso adecuado a la información sobre el medio ambiente de que dispongan autoridades públicas" ${ }^{\prime 58}$.

En otras palabras, la Corte Interamericana, en el caso Claude Reyes y otros vs. Chile, se utilizó de la valoración de las normas narrativas, es decir, la idea de que los valores contenidos en las normas de soft law (Principio 10

56 "Se ha demostrado claramente que los miembros de la comunidad tienen la convicción de que no podrán regresar a su territorio ancestral mientras no obtengan justicia por los hechos de 1986. Andre Ajintoena declaró que después del ataque visitó el área junto con otras personas sólo para recolectar información y sacar fotos del lugar. Una vez que el grupo hubo terminado, algunos de sus integrantes se sintieron enfermos; según el señor Ajintoena, se dieron cuenta de que 'las cosas no estaban bien, no era apropiado, porque de acuerdo con nuestra cultura uno no puede regresar al lugar sin haber hecho arreglos'. Al haber regresado sin 'aplicar las reglas religiosas [y] culturales' - es decir, realizar los rituales mortuorios necesarios y alcanzar reconciliación con los espíritus de quienes fallecieron en el ataque de 1986 (supra párrs. 86.7 a 86.9) - el señor Ajintoena y quienes le acompañaban creían haber ofendido seriamente a esos espíritus y, como consecuencia, empezaron a sufrir enfermedades físicas y psicológicas. Todos los miembros de la comunidad que testificaron ante la Corte expresaron temores similares con respecto a espíritus vengadores, y afirmaron que sólo podrían vivir en la aldea de Moiwana nuevamente si se purificaran primero sus tierras tradicionales" (Caso Moiwana vs. Surinam, cit., par. 113.

57 Caso Claude Reyes y otros vs. Chile, cit., par. 76).

58 “.... En la última Resolución de 3 de junio de 2006 la Asamblea General de la OEA 'instó a los Estados a que respeten y hagan respetar el acceso a la información pública a todas las personas y a promover la adopción de disposiciones legislativas o de otro carácter que fueran necesarias para asegurar su reconocimiento y aplicación efectiva. [...] En igual sentido se debe destacar lo establecido en materia de acceso a la información en la Convención de Naciones Unidas contra la Corrupción y en la Declaración de Río sobre el Medio Ambiente y el Desarrollo' (Caso Claude Reyes y otros vs. Chile, cit., pars. 78-81)". Cf. Principio 10 de la Declaración de Río sobre el Medio Ambiente y el Desarrollo, aprobada en la Conferencia de las Naciones Unidas sobre el Medio Ambiente y el Desarrollo, celebrada del 3 a 14 junio 1992. 
de la Declaración de Río) pueden ayudar a entender el alcance de las normas del hard law (art. 13 de la Convención Americana). Este es el fenómeno de la "emergencia de las normas narrativas", explicada por Erik Jayme como la necesidad de la búsqueda de la comprensión de la ley no sólo desde la comprensión de la función de una norma, sino también desde los valores insertados en ella ${ }^{59}$. En este sentido, las normas de soft law pueden cumplir esta función como la composición de los códigos de conducta, principalmente estructurados por principios como la buena fe, que guían las relaciones jurídicas estructuradas por las normas tradicionales del hard law.

La comunicación intercultural - otro elemento de la posmodernidad se muestra en decisiones marcadas por el "diálogo de las fuentes", es decir, la búsqueda de la solución de un caso no sólo por la aplicación de una única fuente del derecho, sino por la aplicación de la fuente más favorable a la protección los derechos humanos, independientemente de que dicha norma esta presente en un tratado internacional o en la legislación nacional ${ }^{60}$.

En el caso Comunidad Indígena Sawhoyamaxa vs. Paraguay, el diálogo de las fuentes fue utilizado cuando la Corte Interamericana, para decidir sobre la demarcación de las tierras indígenas, analizó las disposiciones de la Convención Americana, de la Convención 169 de la OIT sobre los Pueblos Indígenas y Tribales y las normas constitucionales e infra-constitucionales del derecho interno paraguayo ${ }^{61}$. La misma técnica se utilizó en el caso Saramaka vs. Surinam, cuando la Corte consideró que a pesar de la ausencia en las leyes nacionales surinamesas de cualquier protección de los derechos de propiedad comunal de los pueblos tribales, el Estado demandado había incorporado a su ordenamiento jurídico los Pactos Internacionales de Derechos Civiles y Políticos y de Derechos Económicos, Sociales y Culturales de las Naciones Unidas de 1966, que garantizan los derechos fundamentales (como los derechos de propiedad) a "todos los pueblos" ${ }^{62}$, sin ningún tipo de discriminación. Por lo tanto, la Corte concluyó que la falta de reconocimiento del derecho a la propiedad comunitaria de los cimarrones de Saramaka implicaba violación del art. 21 de la Convención Americana, relacionado al derecho de propiedad ${ }^{63}$.

El diálogo entre los artículos de la Convención Americana y las normas de otros tratados internacionales y de la legislación nacional, con miras a la aplicación de la más beneficiosa al ser humano, está garantizado en el art. 29, b, de la Convención Americana, que no permite ninguna interpretación

59 JAYME, Erik. Identité culturelle et intégration..., cit., 87.

60 V. MAZZUOLI, Valerio de Oliveira. Tratados internacionais de direitos humanos e o direito interno. São Paulo: Saraiva, 2010. p. 129-177; y GOMES, Luiz Flávio \& MAZZUOLI, Valerio de Oliveira. Comentários à Convenção Americana sobre Direitos Humanos: Pacto de San José da Costa Rica, cit., p. 206-207.

61 V. Caso Comunidad Indígena Sawhoyamaxa vs. Paraguay, cit., par. 140.

62 V. art. $1^{\circ}$ del Pacto Internacional de Derechos Civiles y Políticos; y art. $1^{\circ}$ del Pacto Internacional de Derechos Económicos, Sociales y Culturales (1966).

63 V.Caso del Pueblo Saramaka vs. Surinam, cit., par. 93. 
con el objetivo de limitar el alcance de los dispositivos no sólo de la propia Convención, así como de otros tratados internacionales ratificados por Estadoparte.

La técnica de la interpretación pro homine garantizado por cláusulas dialógicas como el art. 29, b, de la Convención, es una alternativa al "monismo clásico", incapaz de diferenciar "normas internacionales por su contenido", una vez que cuando entra en juego la temática de los derechos humanos, hay que ceder el paso al "diálogo" entre las fuentes de protección internacional y nacional con el fin de elegir "la mejor" o "la más beneficiosa norma" que se aplicará al caso que se analiza. ${ }^{64}$

La adopción de un monismo más dialógico ${ }^{65}$ es una gran contribución del sistema interamericano al fortalecimiento del derecho posmoderno, ya que el diálogo entre las fuentes más heterogéneas, de los convenios internacionales a los sistemas nacionales, permite a los "jueces coordinar estas fuentes y escuchar lo que dicen" ${ }^{\prime 66}$.

Otro efecto del "diálogo de las fuentes del derecho" es reforzar la percepción de que el trabajo de los sistemas internacionales de protección de los derechos humanos es complementario y de apoyo al derecho interno. Es decir, el sistema interamericano asume que la primacía de la defensa de los derechos humanos pertenece a los Estados; por lo que los sistemas internacionales y regionales de protección de los derechos humanos en ningún momento son rivales de la jurisdicción de los Estados, pero les ayudan indicando principios que deberán adoptarse a nivel nacional, con miras a la promoción de un propósito común a los Estados de la comunidad internacional: la protección más rápida y efectiva de estos derechos ${ }^{67}$.

Por lo tanto, en la misma forma que el art. 29, b, de la Convención Americana, establece que sus disposiciones deben ceder el paso a la legislación nacional o de otros tratados ratificados por un Estado cuando estos dispositivos resultan más beneficiosos para los seres humanos, también deben aplicar los Estados, en su contexto nacional, las disposiciones de la Convención Americana cuando estas se prueban más efectivas a la protección de los derechos humanos que las normas de la legislación interna ${ }^{68}$. En otras palabras, la utilización por la Comisión y por la Corte Interamericanas del "diálogo de las fuentes del derecho" en temas ambientales es una importante contribución del sistema

64 MAZZUOLI, Valerio de Oliveira. Curso de direito internacional público, cit., p. 90.

65 V. MAZZUOLI, Valerio de Oliveira. Monismo internacionalista dialógico. Revista Jurídica Consulex, Brasília, v. 14, n. 324, p. 50-51.

66 JAYME, Erik. Identité culturelle et intégration..., cit., p. 259.

67 V. AYALA CORAO, Carlos M. Recepción de la jurisprudencia internacional sobre derechos humanos por la jurisprudencia constitucional. Revista del Tribunal Constitucional, n. 6, Sucre, nov. 2004, p. 27.

68 GOMES, Luiz Flávio \& MAZZUOLI, Valerio de Oliveira. Comentários à Convenção Americana sobre Direitos Humanos, cit., p. 209. 
interamericano, no sólo a la solución de casos a nivel internacional, pero sobre todo a las relaciones jurídicas internas ya que el estudio y el análisis de dichas técnicas interpretativas debería proporcionar un incentivo a los Estados Partes de la Convención sobre la adopción en sus respectivos sistemas jurídicos de actitudes más eficaces para la solución de conflictos y la protección del medio ambiente y de los derechos humanos.

\section{CONCLUSIONES}

La protección jurídica del medio ambiente a nivel internacional, garantizado por los principios de la Declaración de Estocolmo sobre el Medio Ambiente Humano (1972) y la Declaración sobre el Medio Ambiente y el Desarrollo en Río de Janeiro (1992), está estructurada por un sistema de conferencias que, si por una parte, permite la aceleración de la entrada en vigor de sus decisiones a través de la adopción de normas de soft law, por otra, no tiene el poder de imponer sanciones a los Estados incumplidores de tales normas. Sin embargo, desde otro ángulo, las Declaraciones de Estocolmo y Río permiten interrelaciones entre las cuestiones ambientales y cuestiones de derechos humanos que ya son amparadas por sistemas de protección más desarrollados.

Así, la percepción de que el acceso a un ambiente "saludable", "equilibrado" y "decente" es una forma de protección de los derechos civiles y políticos (como el derecho a la información, la participación política y el desarrollo de medidas legales de protección), o, por otra parte, de garantía de los derechos económicos, sociales y culturales (como los derechos al desarrollo y el acceso a la atención médica), ha hecho con que cuestiones ambientales sean incorporadas a los asuntos relacionados con la protección de los derechos humanos de la primera dimensión. Por lo tanto, la inclusión de las cuestiones ambientales en el sistema interamericano sólo es posible mediante la vinculación de estos a los dispositivos de la Declaración Americana de los Derechos y Deberes del Hombre y de la Convención Americana sobre Derechos Humanos.

Hay que se reconocer que la estricta observancia de las disposiciones de la Convención Americana para que una cuestión ambiental sea introducida a la sistemática de la Comisión y de la Corte Interamericanas es un obstáculo a la celeridad de la protección internacional del medio ambiente. Sin embargo, también hay que señalar que en la actual estructura normativa internacional, marcada por la debilidad o por normas de "eficacia relativa" de protección del medio ambiente por si mismo, la protección ambiental por la vía refleja o indirecta se demuestra como importante ruta de maduración y de mejora de los mecanismos de protección no solo del medio ambiente, sino también de los derechos humanos. 
Sin embargo, el sistema interamericano no puede ser visto como una tabla de salvación a la protección del medio ambiente y de los derechos humanos, en sustitución del papel de los Estados Partes de la OEA. Por el contrario, su papel es complementario a los Estados, que tienen, estos sí, la primacía de la defensa de los derechos humanos. Por lo tanto, el propósito del sistema interamericano es inducir o fomentar conductas internas compatibles con el objetivo común de la comunidad internacional: la mejora de las políticas destinadas a garantizar los derechos fundamentales.

En este sentido, el estudio de la jurisprudencia del sistema interamericano sobre el acceso a un medio ambiente sano y sus interconexiones con la garantía de los derechos fundamentales no sólo permite una mejor comprensión del alcance de las cuestiones ambientales, pero especialmente indica que las técnicas interpretativas de la Comisión y de la Corte Interamericanas contribuyen a la mejora de la protección del medio ambiente y de los derechos humanos en el ámbito interno de los Estados Partes.

\section{BIBLIOGRAFÍA}

ANKERSEN, Thomas T.; RUPPERT, Thomas K. Defending the polygon: the emerging human right to communal property. Oklahoma Law Review, v. 59, n. 4 (2006), p. 681 757.

AYALA CORAO, Carlos M. Recepción de la jurisprudencia internacional sobre derechos humanos por la jurisprudencia constitucional. Revista del Tribunal Constitucional, n. 6, Sucre, nov. 2004, p. 27.

BOYLE, Alan. Human rights and the environment: a reassessment. UNEP Human Rights and Environment, 2010, p. 1-39.

CANÇADO TRINDADE, Antônio Augusto. Direitos humanos e meio-ambiente: paralelo dos sistemas de proteção internacional. Porto Alegre: Sérgio Antônio Fabris Editora, 1993.

CANÇADO TRINDADE, Antônio Augusto. Os direitos humanos e o meio ambiente. In: SYMONIDES, Janusz (Org). Direitos humanos: novas dimensões e desafios. Brasília: UNESCO Brasil/Secretaria Especial dos Direitos Humanos, 2003, p. 162-203.

CEDERSTAV, Anna K; BARANDIARÁN G., Alberto. La Oroya cannot wait. Lima: Sociedad Peruana de Derecho Ambiental, setembro de 2002.

ENGLE, Eric. Third party effect of fundamental rights (Drittwirkung). Hanse Law Review, v. 5, n. 2, Bremen: Hanse Law School, p. 165-173.

FIX-ZAMUDIO, Hector. Protección jurídica de los derechos humanos. México: Comisión Nacional de Derechos Humanos, 1991.

GARFIELD, Seth. A nationalist environment: indians, nature and the construction of the Xingu National Park in Brazil. Luso-Brazilian Review, v. 41, n. 1, Madison: University of Wisconsin Press, 2004, p. 139-167.

GOMES, Carla Amado. Textos dispersos de direito do ambiente. Lisboa: Associação Acadêmica da Faculdade de Direito, v. III, 2010. 
GOMES, Luiz Flávio; MAZZUOLI, Valerio de Oliveira. Comentários à Convenção Americana sobre Direitos Humanos: Pacto de San José da Costa Rica. 3. ed. rev., atual e ampl. São Paulo: Revista dos Tribunais, 2010.

HUMAN RIGHTS WATCH. World Report 1994: Events of 1993. New York: Human Rights Watch, 1994.

JAYME, Erik. Identité culturelle et intégration: Le droit international privé post-moderne. Recueil des Cours, v. 251 (1995), p. 9-267.

KISS, Alexandre; SHELTON, Dinah. Guide to international environmental Law. Koninlijke Brill, NV, Leiden: Martinus Nijhoff Publishers, 2007.

KISS, Alexandre; SHELTON, Dinah. Judicial handbook on environmental Law. Nairobi: United Nations Environment Programme, 2005.

MAGRAW, Daniel Barstow; BAKER, Lauren. Globalization and communities: community-based property rights and prior informed consent. Denver Journal of International Law and Policy, v. 35, ns. 3 e 4, 2007/2008, p. 413-428.

MAYZOLER, Marcel; ROUDART, Laurence. História das agriculturas no mundo: do neolítico à crise contemporânea. Trad. Cláudia F. Fallhuh Balduino Ferreira. São Paulo: Editora Unesp; Brasília: NEAD, 2010.

MAZZUOLI, Valerio de Oliveira. Curso de direito internacional público. 5. ed. rev., atual. e ampl. São Paulo: Editora Revista dos Tribunais, 2011.

. Monismo internacionalista dialógico. Revista Jurídica Consulex, Brasília, v. 14, n. 324 , p. 50-51.

. Tratados internacionais de direitos humanos e o direito interno. São Paulo:

Saraiva, 2010.

. Direito internacional privado: curso elementar. Rio de Janeiro: Forense, 2015, p. 199-212.

; IRIGARAY, Carlos Teodoro Hugueney (Orgs). Novas perspectivas do direito ambiental brasileiro: visões interdisciplinares. Cuiabá: Cathedral, 2009.

; TEIXEIRA, Gustavo de Faria Moreira. Greening the Inter-american human rights system. L'Observateur des Nations Unies, v. 33 (2013), p. 299-313.

MITCHELL, Ronald B. Problem structure, institutional design, and the relative effectiveness of international environmental agreements. Global Environmental Politics, v. 6, n. 3 (2006), p. 72-89.

OSOFSKY, Hari M. Inuit petition as a bridge? Beyon dialectics of climate change and indigenous people's rights. American Indian Law Review, v. 31 (2007), p. 675-698.

PIOVESAN, Flávia. Direitos Humanos: Desafios e perspectivas contemporâneas. Revista do Instituto de Direito Constitucional e Cidadania, ano I, v. 1, abril 2005, p. 49-76.

RODRÍGUEZ-PINZÓN, Diego; MARTíN, Claudia. A proibição de tortura e maus-tratos pelo sistema interamericano: um manual para vítimas e seus defensores. Trad. Regina Vargas. Ginebra: World Organization Against Torture (OMTC), 2006.

SANDS, Philippe (ed.).Greening international law. London: Earthscan Publications Limited, 1993. 
SHELTON, Dinah. Environmental rights and Brazil's obligations in the inter-american human rights system. George Washington International Law Review, vol. 40 (2008), p. 733-777.

SHELTON, Dinah. Human rights, health and environmental protection: linkages in Law and practice. Health and human rights working paper series, $\mathrm{n}^{\circ}$ 1.Ginebra: World Health Organization, 2002, p. 3-22.

SHIVA, Vandana. Biopirataria: a pilhagem do conhecimento. Trad. Laura Cardellini Barbosa de Oliveira. Petrópolis: Vozes, 2001.

SOARES, Guido. Direito internacional do meio ambiente: emergências, obrigações e responsabilidades. São Paulo: Atlas: 2001.

SOUSA SANTOS, Boaventura de. Uma concepção multicultural de direitos humanos. Revista Lua Nova, São Paulo, v. 39 (1997), p. 105-201.

SPILLER, Paula. The La Oroya case: the relationship between environmental degradation and human rights violation. Human rights brief, v. 18, issue 1, Washington D.C: American University of Washington College of Law, fall 2010, p. 19-23.

TEIXEIRA, Gustavo de Faria Moreira. O greening no sistema interamericano de direitos humanos. Curitiba: Juruá, 2011.

VARVARIGOS, Dimitrios. Environmental degradation, longevity and the dynamics of economic development. Environmental and Resource Economics, v. 46, issue 1 (2010), p. 59-73. 


\title{
A Extrafiscalidade como Instrumento de Proteção do Meio Ambiente Ecologicamente Equilibrado
}

\author{
Extraficality as a Means to Protection of an Ecologically Balanced Enviroment
}

\section{CAROLINA SCHROEDER ALEXANDRINO}

Advogada, Bacharel em Direito - Ciências Jurídicas e Sociais pela Unisinos, Bacharel Laureada, premiada pelo primeiro lugar no Curso de Direito da Unisinos e como aluna destaque entre os formandos. Possui especialização em Advocacia Tributária pela ESA/RS, curso de extensão certificado pela Instituição Faculdade IDC: Extensão em Direito Previdenciário, curso de extensão certificado pelo escritório especializado em Consultoria, Empresarial Scaliilli.fmv: Gestão de Crise, Falências e Recuperação Judicial.

\section{MARCIANO BUFFON}

Doutor em Direito com ênfase em Direito do Estado pela Unisinos, com período de pesquisa na Universidade de Coimbra, Mestre em Direito Público, Advogado Tributarista, com especialização em Direito Empresarial, Professor de Direito Tributário na Unisinos (São Leopoldo/RS) e em cursos de especialização em Direito Tributário em outras instituições, Professor no Programa de Pós-Graduação em Direito da Unisinos, Sócio/Consultor jurídico-fiscal do escritório Buffon \& Furlan Advogados Associados, Membro do Conselho Técnico de Assuntos Tributários, Legais e Financeiros da Federação das Indústrias do Estado do Rio Grande do Sul - Fiergs.

Submissão: 11.05 .2015

Decisão Editorial: 21.07.2015

Comunicação ao autor: 21.07.2015

RESUMO: 0 presente estudo busca demonstrar como a tributação, com finalidade não fiscal, pode consubstanciar-se em um meio eficaz de proteção do meio ambiente ecologicamente equilibrado. Nesse escopo, analisa-se a extrafiscalidade, que objetiva a realização de valores que ultrapassam a mera arrecadação de tributos; como instrumento de preservação ambiental dentro de uma sociedade reflexiva, de risco. Para tanto, a extrafiscalidade é demonstrada em dois principais segmentos: a) pelos impostos e b) por meio dos benefícios fiscais. Assim, traça-se um elo entre o Direito Tributário e o Direito Ambiental a partir do estudo da extrafiscalidade como ferramenta de proteção do meio ambiente.

PALAVRAS-CHAVE: Extrafiscalidade; tributação; direito ambiental; gerenciamento dos riscos; políticas públicas. 